\title{
MARINE GEOID UNDULATION ASSESSMENT OVER SOUTH CHINA SEA USING GLOBAL GEOPOTENTIAL MODELS AND AIRBORNE GRAVITY DATA
}

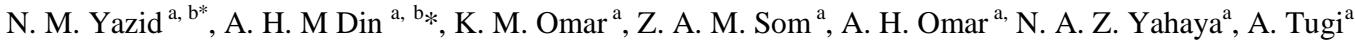 \\ ${ }^{a}$ Geomatic Innovation Research Group (GIG), Faculty of Geoinformation and Real Estate, Universiti Teknologi \\ Malaysia, 81310 Johor Bahru, Johor, Malaysia. \\ ${ }^{\mathrm{b}}$ Geoscience and Digital Earth Centre (INSTEG), Universiti Teknologi Malaysia, 81310 Johor Bahru, Johor, \\ Malaysia. \\ *amihassan@utm.my
}

KEY WORDS: Global Geopotential Model, Geoid Undulations, Airborne Gravity, Gravity Missions

\begin{abstract}
:
Global geopotential models (GGMs) are vital in computing global geoid undulations heights. Based on the ellipsoidal height by Global Navigation Satellite System (GNSS) observations, the accurate orthometric height can be calculated by adding precise and accurate geoid undulations model information. However, GGMs also provide data from the satellite gravity missions such as GRACE, GOCE and CHAMP. Thus, this will assist to enhance the global geoid undulations data. A statistical assessment has been made between geoid undulations derived from 4 GGMs and the airborne gravity data provided by Department of Survey and Mapping Malaysia (DSMM). The goal of this study is the selection of the best possible GGM that best matches statistically with the geoid undulations of airborne gravity data under the Marine Geodetic Infrastructures in Malaysian Waters (MAGIC) Project over marine areas in Sabah. The correlation coefficients and the RMS value for the geoid undulations of GGM and airborne gravity data were computed. The correlation coefficients between EGM 2008 and airborne gravity data is 1 while RMS value is 0.1499.In this study, the RMS value of EGM 2008 is the lowest among the others. Regarding to the statistical analysis, it clearly represents that EGM 2008 is the best fit for marine geoid undulations throughout South China Sea.
\end{abstract}

\section{INTRODUCTION}

\subsection{Background of the study}

Geoid generally can be defined as a level surface and also as being everywhere perpendicular to gravity (Talone et al., 2014). The shape of the geoid is actually relatively close to the shape of an ellipsoid with an equatorial radius $21.4 \mathrm{~km}$ longer than the polar radius (Hughes and Bingham, 2008). Therefore, the global geoid undulations from EGM 2008, ITG GOCE 02, AIUB CHAMP 03s and ITG GRACE 02s are employed in this study for data derivation and verification.

Global Geopotential Model (GGM) can be described as the mathematical model that clarifies gravitational potential in a spectral domain by using spherical harmonic expansions. GGM has been published by the International Center for Global Earth Models (ICGEM).Besides, these models can provide the medium and long wavelength part of a gravimetric geoid in the resolution and accuracy aspect (Sadiq \& Ahmad, 2009)and comprise data mainly from CHAMP (Reighber et al., 1999) and GRACE (Reighber et al., 2000) satellite gravity missions. Generally, GGMs are comprised of two main types, so called satellite only GGM and combined GGM for the determination of global, regional and local geoid models. According to Sulaiman (2016), the development of GGM consists of the combining of two existing GGMs called tailored GGMs but these GGMs do not published by ICGEM.
Thus, this study is concerted on the main types of GGMs published by ICGEM (Satellite-only and Combined). Satellite-only GGMs are derived from the assessment of satellite-based gravity observations (CHAMP and GRACE), or combined with other satellite missions such as Laser Geodynamic Satellite (LAGEOS), European Remote Sensing Satellite (ERS) and Geodetic Satellite (GEOSAT). Figure 1 represents a sample of spectral evaluations of satellite-only and combined GGM, which is compared to the most recent combined model. Each spectral evaluation model consists of the signal amplitudes per degree of the model and the differences in amplitudes with the recent combined model.
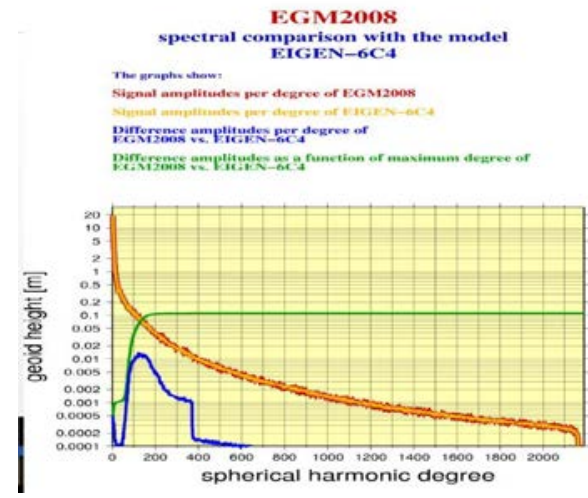

Figure 1. Spectral Comparison of EGM 2008 with the Model EIGEN-6C4 (ICGEM, 2015) 
Featherstone and Rummel (2002) emphasized that the accuracy of satellite-only GGMs are weak at the higher degree of coefficients due to power decay of the gravitational field with altitude, incomplete tracking of satellite orbits using ground stations, less precise modeling of non-Earth gravity field induced satellite motion, such as atmospheric drag and third body influences, and poor coverage of sampling global gravity fields.

Combined GGMs are derived from combinations of satelliteonly GGMs with terrestrial gravity data (Land and Marine), gravity anomaly for satellite altimetry, airborne gravimeter, topography and bathymetry. By combining all the gravity data, some of the limitations on the higher degree expansion can be diminished; however the errors in terrestrial data still remain. Generally, combined GGMs provide better fits to the terrestrial gravity data than satellite-only models. Besides, these combinations leads towards the best approximation of the Earth's gravitational field.(Sadiq \& Ahmad, 2009).In this study, both (combine and satellite-only) GGMs are required. GGMs will be used to improve the deficiencies in land and marine gravity data, while satellite only GGMs will be used in the LSMS formula in the determination of precise, seamless geoid models

Moreover, the medium to wavelength geoid signal is the main component of the geoid, $N$ and its value can be assessed from GGM. It can be expressed in terms of a set of spherical harmonic coefficients up to a certain degree and order. The localised geoid undulations can be achieved when a best fit GGM is chosen for a certain area (Sadiq \& Ahmad, 2009)

\subsection{Geoid Undulations of Global Geopotential Model (GGM)}

The geoid is an equipotential surface of the Earth's gravity field that is closely associated with the location of the mean sea surface (Picot et al., 2003). Geoid also can be defined as a level surface defined as being everywhere perpendicular to gravity (Talone et al., 2014). Therefore, in a motionless ocean, the sea surface would be everywhere parallel to the geoid (Talone et al., 2014). The shape of the geoid is actually relatively close to the shape of an ellipsoid with an equatorial radius $21.4 \mathrm{~km}$ longer than the polar radius (Hughes and Bingham, 2008).

This ellipsoid gives the plane normal to the local effective gravity, which is no more than the vector addition of the Earth's gravity acceleration and the Earth's centripetal acceleration (a function of latitude, associated with the rotation of the Earth around its axis) (Talone et al., 2014). However, the geoid may locally depart from this ellipsoid by up to $100 \mathrm{~m}$ because of regional changes in the gravitational field (Hughes and Bingham, 2008).

The global geoid heights also can be extracted from the International Center for Global Earth Model (ICGEM) conducted spectral evaluations by comparing the existing GGMs with the new combined GGM such as ITGGRACE 02s, EGM 2008, ITG-GOCE 02s and AIUB CHAMP 03s. From the current (Oct 2012) global evaluation, EGM 2008 is the best fit GGM for a combined solution. However, the results from global evaluation should not be used directly as a suitable GGM model for the determination of localised geoid model. (Kiamehr, 2006).

Global evaluation results can be used as a guide for selecting the most appropriate GGM for localised geoid undulations model determination. Therefore, in the determination of localised geoid undulations models, the localised evaluation of the most suitable model should be performed. Thus, this study will assist to ascertain the most feasible GGM which best matches for the local marine geoid undulations covering South China Sea.

\subsection{Airborne Gravity Data}

In this study, the accuracy of GGMs are evaluated with airborne gravity data under the Marine Geodetic Infrastructures in Malaysian Waters (MAGIC) project in Sabah. This project involves some recent (2014-2015) airborne gravity surveys undertaken by the Department of Survey and Mapping Malaysia (DSMM) (JUPEM, 2014/2015).

Airborne gravity data from previous field campaign carried out in 2002-2003 over land area in Sabah has been combined with the present marine airborne gravity data to provide a seamless land-to-sea gravity field coverage (JUPEM, 2003). The airborne gravity survey database for land and marine areas of Sabah is considered complete and has been compiled in ArcGIS geo-database format. Some geological inferences also has been presented to initiate further research on the application of gravity field in marine geology and geophysics.

Airborne gravimetry is an effectual implement for mapping local gravity fields using a combination of airborne sensors, aircraft and positioning system. It is appropriate for gravity surveys over difficult terrains and areas mixed with land and ocean. The development of airborne gravimetry has been made possible by the employment of the kinematic Global Positioning System (GPS) technique as well as improvement in airborne gravity acceleration sensor system. The best accuracy of the airborne gravimetry is $1-2 \mathrm{mGal}$ at $5 \mathrm{~km}$ resolution for fixed-wing aircraft (Forsberg et al, 1999 and Olesen et al, 2007).

Gravity measurements from an airborne are performed on the background of inertial disturbing accelerations which are caused by the vehicle's dynamics (Childers et al, 1999). The value of inertial accelerations may be several hundred thousand times as high as the valid signal and can overlap with it in the spectral frequency domain (Sokolov, 2011). The analysis of spectral characteristics of marine inertial acceleration allows the methods of low-frequency filtering to be used in practice to eliminate the effect of vertical disturbing accelerations during marine survey (Zheleznyak ,2002).Thus, this purpose can be achieved with the employment of coprocessing of gravimetric data and navigation information (Stepanov et al, 2005). 
The assessment of marine geoid undulations by using GGMs; EGM 2008, ITG-GRACE 2010s, ITG-GOCE 02s and AIUB CHAMP 03s is useful for the determination of the best GGM for Malaysian seas. This can be utilised for further research in order to determine the localised marine geoid undulations for Malaysian seas. Nevertheless, the significance of the GGM are useful for the determination of global geoid undulations and gravity anomaly.

\subsection{RESEARCH APPROACH}

\subsection{Study Area.}

The area under study is bounded between latitude $1^{\circ} \mathrm{N}$ to $9^{2} \mathrm{~N}$ and longitude $109^{\circ} \mathrm{E}$ to $120^{\circ} \mathrm{E}$ as represented in Figure 2. This study area is focused on South China Sea. The study area is chosen based on the airborne gravity data provided by DSMM covering this area.

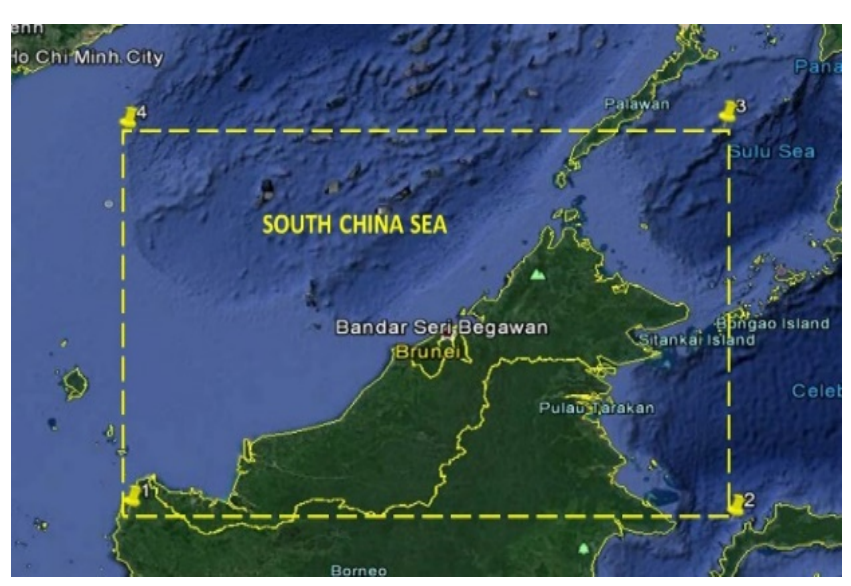

Figure 2. The map of the study area

2.2

Data Preparation and Processing

Currently, there are more than 50 geopotential models available at the International Center for Global Gravity Earth Models (ICGEM) and an open source for the global scientific community at http://icgem.gfz-potsdam.de/ICGEM/. In this study, discussion is emphasised on the GGM from satelliteonly and the combined. Four (4) GGM have been selected which are EGM 2008, ITG-GRACE 2010s, ITG-GOCE 02s and AIUB CHAMP 03s as shown in Table 1.

This selection is based on necessity for evaluation so that a match could be achieved in both medium and long wavelength with the ground truth data (Sadiq \& Ahmad, 2009). Among these EGM 2008 is the developed combined GGM with very high degree of spherical harmonic expansion which is up to 2190 degree. EGM 2008 is the only combined model that is selected in this study. This is based on the earlier research,(Pavlis et al., 2008; Sadiq \& Ahmad, 2009; Yi \& Rummel, 2014) where EGM 2008 is the most comprehensive representation and the highest resolution of the Earth's gravitational field.

\begin{tabular}{|c|c|c|c|}
\hline Model & Year & Degree & Data \\
\hline EGM 2008 & 2008 & 2190 & S(Grace),G,A \\
\hline $\begin{array}{c}\text { AIUB- } \\
\text { CHAMP03S }\end{array}$ & 2010 & 100 & S(Champ) \\
\hline $\begin{array}{c}\text { ITG- } \\
\text { Grace2010s }\end{array}$ & 2010 & 180 & S(Grace) \\
\hline ITG-Goce02 & 2013 & 240 & S(Goce) \\
\hline
\end{tabular}

Table 1. The list of GGMs for Evaluations

Nevertheless, the computation of GGMs is compressed with a set of fully normalized spherical harmonic coefficients that represent the Earth's gravitational potential outside relevant masses. Each geopotential model will provide the information regarding to the Newtonian gravitational constants $(G)$ and the mass of the earth $(\mathrm{M})$, normal gravity on the surface of the reference ellipsoid $(\gamma)$, a reference radius $(R)$, fully normalized Stokes' Coefficients for each degree $n$ and order $m\left(\overline{C_{n m}}\right.$ and $\left.\overline{S_{n m}}\right)$ and its respective standard errors $\left(\sigma \overline{C_{n m}}\right.$ and $\left.\sigma \overline{S_{n m}}\right)$. Thus, the geoid heights and gravity anomalies can be computed based on these equations.

Therefore, to compute the geoid undulations regarding to the GGMs data, Equation 1 is referred.

$$
\begin{aligned}
& \Delta N_{G G M}=\frac{G M}{r \gamma} \sum_{n=2}^{m}\left(\frac{\bar{q}}{\bar{r}}\right)^{n} \sum_{m=0}^{n}\left(\bar{C}_{n m} \cos m \lambda+\bar{S}_{n m} \sin m \lambda\right) \bar{P}_{n m}(\cos \theta) \\
& \text { Where } \Delta N_{G G M} \quad=\text { the geoid heights derived from the global }
\end{aligned}
$$

The spatial resolution of geoid heights provided by GGM on the Earth's surface is determined by the maximum number of complete harmonic expansions (max).

The geoid undulations from GGMs are computed by EGMlab1 software programmed by Mehdi Eshagh and Ramin Kiamehr from Division of Geodesy, Royal Institute of Technology, Stockholm, Sweden in year 2012. This program computed the anomalies quantities such as geoidal height, gravity anomaly east-west and north-south components of deflection of verticals using GGMs.

There are some considerations must be counted while making the assessment of the GGMs with the ground truth data (Sadiq \& Ahmad, 2009):

i. The scale difference between the GGM and adopted reference ellipsoid parameters, eg. Earth-gravity mass constant GGM and semi-major axis, etc. provide towards the difference in the results. 
ii. $\quad$ The collected terrestrial gravity data may have medium and long wavelength errors and GGM derived quantities are relatively less prone to this type of error as mentioned by Heck (1990).

As mentioned by Kiamehr (2006) and Sulaiman (2016) the assessment of the global geoid undulations should not be directly employed to nominate the most appropriate GGM for the determination of a geoid model. This due to the global statistics does not essentially signify the valid information about the region. In finalising the most suitable GGM for the localised geoid undulations modelling, the following approaches are commonly depleted:

i. Analysing the differences between the geoid undulations from airborne gravity data and GGM.

ii. Compute the root mean square (RMS) different between the geoid undulations from GGM and airborne gravity data.

Thus, the assessment of the mentioned approach is analysed in order to ascertain the most appropriate GGM suited for Malaysian seas Then, four (4) GGM were evaluated as represented in Table 1 . The assortment criteria are generally based on which of the GGMs is the closest fit to the geoid undulations from airborne gravity data.

Normally, geoid undulations are derived from GGM based on Equation 1. The assessment of the best fit GGM for local use are required to compare the derived geoid undulations from GGMs with the airborne gravity data. Therefore, the best fit GGM is determined based on the lowest RMS in the statistical analysis of the geoid undulations residual. The geoid undulations residual is computed by using Equation 2 .

$\Delta N_{\text {residual }}=N_{\text {GGM }}-N_{\text {airborne }}$

Where $\Delta N_{\text {residual }}$ is the residual geoid undulations, $N_{\text {GGM }}$ is the geoid undulations from GGM and $N_{\text {airborne }}$ is the geoid undulations from the airborne gravity data.

Subsequently, the RMS different for the $\Delta N_{\text {residual }}$ was computed based on Equation 3:

$R M S=\sqrt{\frac{\sum_{l=0}^{H i}\left(\Delta N_{\text {rsetdund }}\right)^{2}}{n}}$

Where $\mathrm{n}=$ the total number of points

Thus, the lowest RMS different for the assessment of $N_{G G M}$ with $N_{\text {airborne }}$ indicate the best fit GGM for the South China Sea.

\subsection{Marine Geoid Undulations from Airborne Gravity Data}

The geoid undulations from GGM are evaluated with airborne gravity data from DSMM. The GGM that present closest statistical fit to the ground truth data can be implicit as the most suitable model to adopt for the determination of local marine geoid undulation for South China Sea. Nevertheless, the best fit of the GGM with airborne gravity data is the lowest standard deviation of the differences and the lowest R.M.S but not the best model (Rudriguez-Caderot et al., 2006) Figure 3 represents the airborne track covering the South China Sea. Figure 4 corresponds to the map of the airborne gravity data covering the South China Sea.

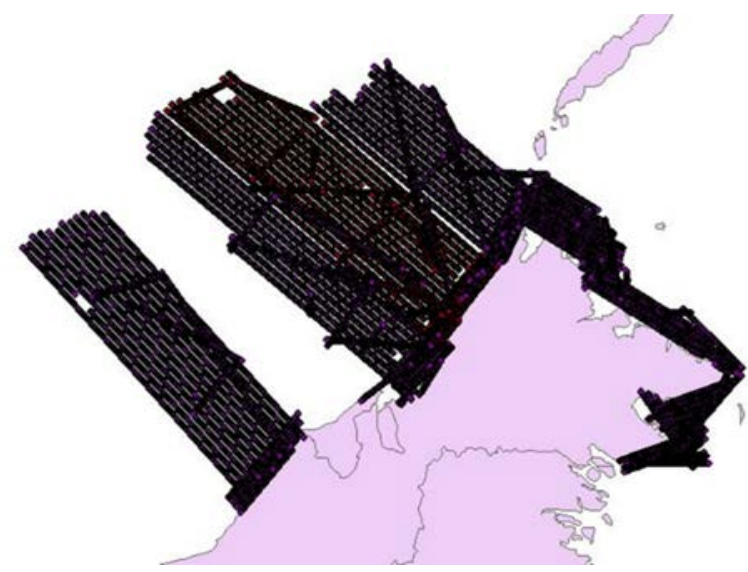

Figure 3. Airborne track in Sabah

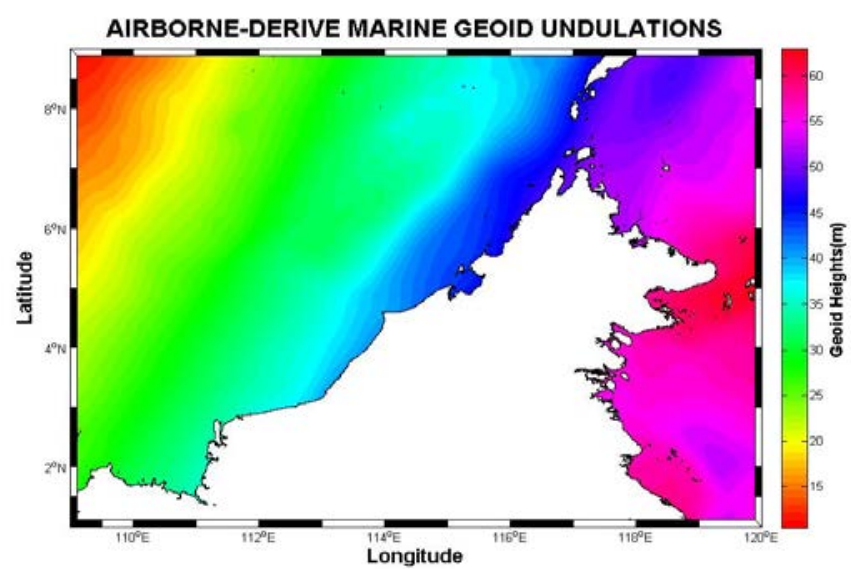

Figure 4: Airborne-derived marine geoid map covering South China Sea

\subsection{RESULTS AND DISCUSSION}

\subsection{Global Geopotential Models (GGMs); EGM 2008, ITG-GRACE 2010s, ITG-GOCE 02s and AIUB CHAMP03s}

The geoid undulations from GGMs have been extracted for the Malaysian region covering the South China Sea on $0.25^{\circ}$ by $0.25^{\circ}$ regular grids. These extractions are vital in order to evaluate the geoid undulations from GGM and airborne gravity data. The ICGEM format accommodates the GGM in terms of spherical harmonic coefficients, the ocean and atmosphere tides. Figure 5 illustrates the EGM 2008, ITGGRACE 2010s, ITG-GOCE 02s and AIUB CHAMP 03s. These evaluations will assist the clarification of the best GGM that is merged to the South China Sea. Based on Figure 5, it seems very similar performances in terms of mapping. However, there are differences for each GGM as explained in Figure 6, 7 and Table 2. 

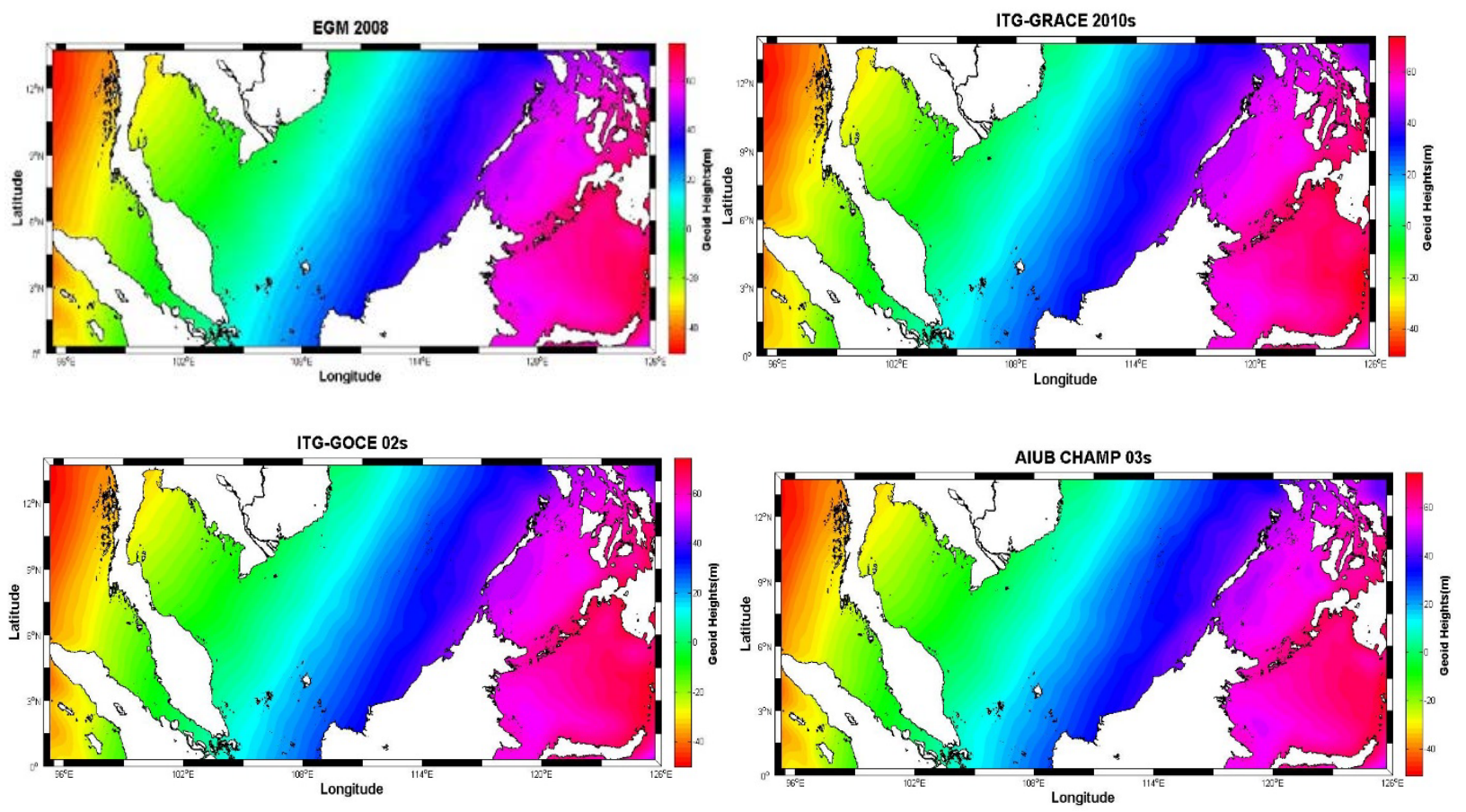

Figure 5. The geoid undulations from GGM (EGM 2008, ITG-GRACE 2010s, ITG-GOCE 02s and AIUB CHAMP 03s)

\subsection{Comparisons of Marine Geoid Undulations from GGMs and Airborne Gravity Data}

The information on the top rankings of GGMs based on Root Mean Square (RMS) difference between GGM compared to airborne gravity data from Department of Survey and Mapping Malaysia (DSMM) as display in Table 2. This assessment is comprised of well distributed 130 selected points covering the South China Sea.

As mentioned in Section 1.1, the advantage will be achieved in the localised geoid undulations modelling process when a best fit GGM is chosen for a certain region. This will contribute towards the reduction of the geoid entailed from regional integration of Stoke's formula or other amendments (Sadiq \& Ahmad, 2009). Besides, some errors contained in GGMs which are distinguished with the evaluation from airborne gravity data.

The correlation between the airborne gravity data and the GGMs as shown in Figure 6. Thus, EGM 2008 represents the highest correlation compared to the other GGMs. The correlation between EGM 2008 and airborne gravity data is equal to 1 . Thus, it can be described as the relationship or association between EGM 2008 and airborne gravity data is so related with each other. Nevertheless, ITG-GRACE 2010s is the lowest correlation coefficients compared to the others.
Therefore, it is the most suitable GGM to adopt for the determination of local marine geoid undulations for Malaysian Sea. However, the verification towards the assessment of GGMs and airborne gravity data need to be discussed using statistical analysis.

The value of RMS for GGM both combined and satellite-only fluctuates are represented on Table 2. Nevertheless, the value still can be recognized. The range value of RMS is $0.1499 \mathrm{~m}$ to 0.6157 for combined and satellite-only. EGM 2008 had the lowest RMS value compared to ITG-GRACE 2010s, ITGGOCE 02s and AIUB CHAMP 03s. AIUB CHAMP03s represents the highest which is out to $0.6157 \mathrm{~m}$ compared to the EGM 2008 which is only 0.1499. EGM 2008 is from the combined GGM that the accuracy of the combined GGM may be combined in terms of less omission errors in the characteristics of the models, and not necessarily in the improvement in low frequency measurements (Amos \& Featherstone, 2003).

Besides, EGM 2008 is also from the combined GGMs which generally provide better fits to the gravity data than the satellite-only models (Sadiq \& Ahmad, 2009). Nevertheless, it is conceivable that the satellite-only GGMs are more precise than the combined GGMSs because the later have infectivity from long and medium wavelength errors in the terrestrial gravity-data. 

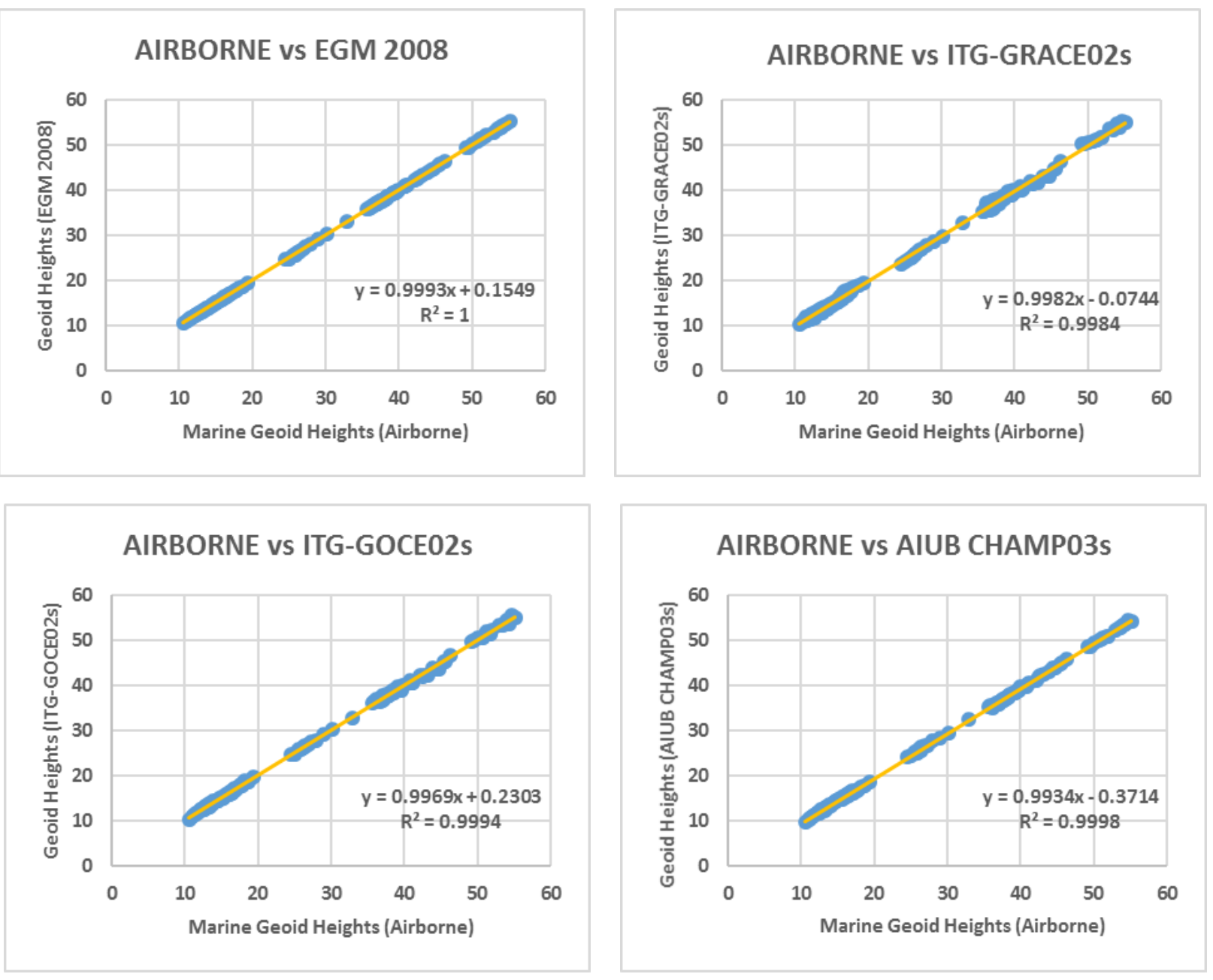

Figure 6. The correlation between the airborne gravity data and the GGMs (EGM 2008, ITG-GRACE 2010s, ITG-GOCE 02s and AIUB CHAMP 03s

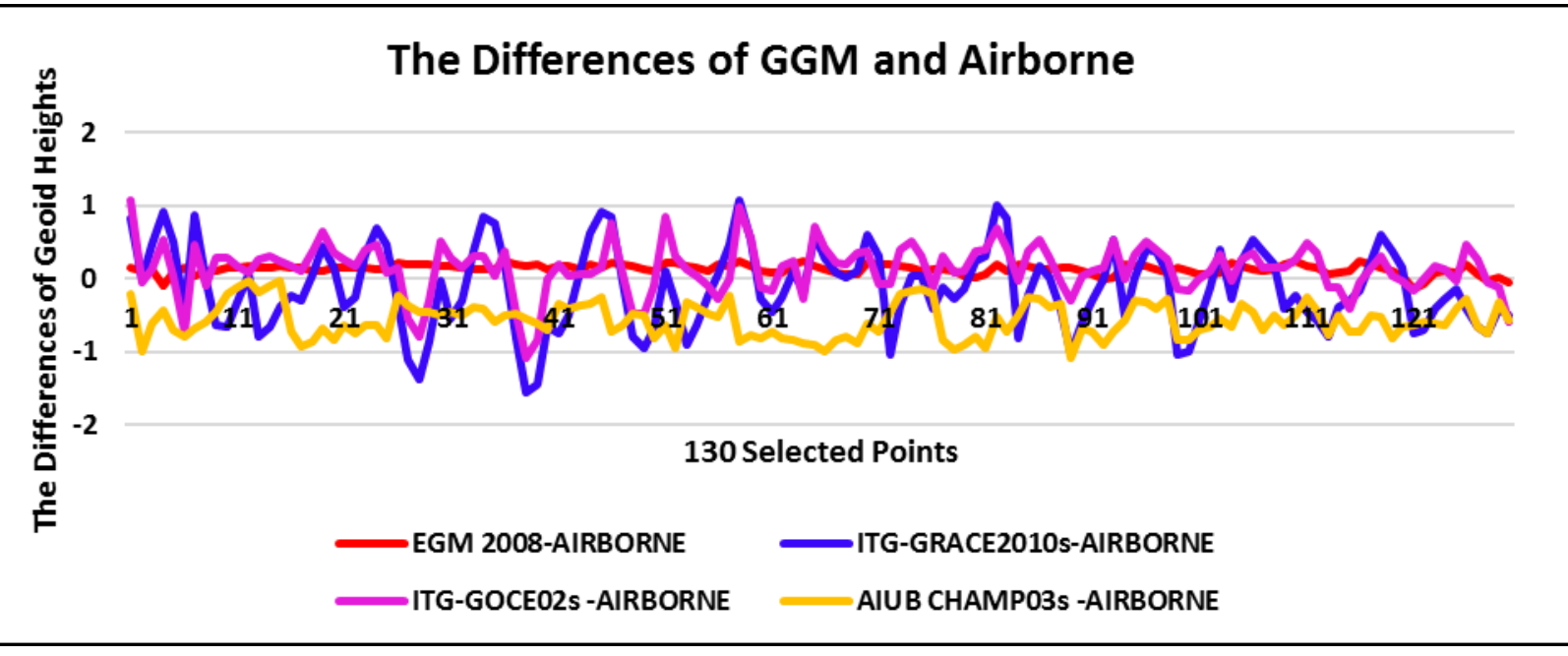

Figure 7. The difference of GGM data (EGM 2008, ITG-GOCE02s, ITG-GRACE2010s and AIUB CHAMP03s) with airborne gravity data 
The International Archives of the Photogrammetry, Remote Sensing and Spatial Information Sciences, Volume XLII-4/W1, 2016 International Conference on Geomatic and Geospatial Technology (GGT) 2016, 3-5 October 2016, Kuala Lumpur, Malaysia

\begin{tabular}{|c|c|c|c|c|}
\hline & EGM 2008 & ITG-GRACE 2010s & ITG-GOCE 02s & AIUB CHAMP 03s \\
\hline Min(m) & 10.79153 & 10.3726 & 10.4963 & 9.8198 \\
\hline Max(m) & 55.2856 & 55.1672 & 55.1193 & 54.1808 \\
\hline Max Difference (m) & 0.2475 & -1.5645 & 1.0779 & -1.0756 \\
\hline RMS (m) & 0.1499 & 0.5694 & 0.3641 & 0.6157 \\
\hline
\end{tabular}

Table 2. Statistic of geoid heights from GGMs compared to airborne gravity data

According to Figure 6, 7 and as shown in Table 2, EGM 2008 have very similar performances with the highest correlation $(1 \mathrm{~m})$, the smallest maximum difference value $(0.2475 \mathrm{~m})$ and the smallest RMS value (0.1499) to airborne gravity data. EGM 2008 also being less than the ITG-GRACE 2010s, ITGGOCE02s and AIUB CHAMP 03s. This is due to the free-air anomalies from airborne ellipsoid heights, converted to orthometric heights by the EGM2008 geoid undulations. Besides, the orthometric heights of the airborne is also referred to the EGM 2008.

Therefore, this provides the high correlation, the smallest maximum differences and the smallest RMS value between EGM 2008 and airborne gravity data. Thus, it clearly represents that EGM 2008 have the best fit for marine geoid undulations throughout the South China Sea. It could be indicated that the ITG GOCE 02, AIUB CHAMP 03s and ITG GRACE 2010s do not fit the region. Thus, it could be sufficient to prove the geoid undulations from EGM 2008 is the best GGM for Malaysian sea. EGM 2008 can be employed to enhance the deficits in the marine gravity data. This statistical results can be corroborated based on earlier studies (Sadiq \& Ahmad, 2009) which is EGM 2008 is the very high degree of spherical harmonic expansion in relation to the new data addition.

Nevertheless, it should be emphasised that the results just a global evaluation test on the selected areas only. Thus, the results from the global evaluation should not be used directly as a suitable GGM model for localised marine geoid determination(Kiahmehr, 2006). This can be verified based on the previous research, the EGM 2008 is the most comprehensive representation and the highest resolution of the Earth's gravitational field currently available. (Yi \& Rummel, 2014) It combines the GRACE satellite gravitational model ITG-GRACE 03s (Mayer-Gürr, 2007) with a global set of an area-mean free-air gravity anomalies given on a 5 arc-minute equiangular grid.

However, in the other regions such as South America, Africa, South-East Asia or China, where the surface gravity data available for the development of EGM 2008 were poor. The RMS differences in this region are on a level of $30 \mathrm{~cm}$. Besides, Sulaiman (2016) conducted a study related to land gravity observation throughout Peninsular Malaysia. Therefore, it is found that EGM 2008 represents the top of the list of GGM for land gravity data with .RMS $0.095 \mathrm{~m}$. Therefore, it can be concluded that EGM 2008 is the most suitable GGM for the marine and land regions for Malaysian Seas.

\section{CONCLUSIONS}

As such this assessment of ground truth data with GGM has presented important information towards the best fit GGM for subsequent gravimetric geoid. Based on the statistical analysis, this study can be concluded that EGM 2008 is the best fitting GGM which has the lowest RMS and standard deviation latter the assessment with the airborne gravity data. The statistical results are used to advocate the EGM 2008 in all aspects. The reasons of this due to the high degree of spherical harmonic expansion in relation to the addition of new data. If the localised geoid undulations based on the satellite-only GGM, ITG-GOCE 02s could be appropriate to replace EGM 2008. Since, ITG-GOCE 02s is the second best GGM for this study.

Besides, EGM 2008 also represents the highest correlation coefficients compared to the others. However, ITG-GRACE 2010s represent the worst correlations coefficients with airborne gravity data Therefore, it can be concluded that EGM 2008 is the best matches of GGM with the local marine geoid undulations of the South China Sea. It is highly recommended that the assessment of GGM with airborne gravity data can assist towards the determination of local marine geoid undulations by using the latest technology. For example the satellite radar altimeter. The combination between the satellite altimeter and satellite gravity missions data could grant the high dense data.

\section{ACKNOWLEDGEMENTS}

Highly acknowledged to the Department of Survey and Mapping Malaysia (DSMM) for the airborne gravity data under the Marine Geodetic Infrastructures in Malaysian Waters (MAGIC) Project over marine areas in Sabah year 2015. High appreciation to ICGEM for providing an open access GGM data.

We also acknowledge Dr. Ramin Kiamehr for sharing his EGMLAB software, which is available from Mr. Zainal Abidin Md Som. Furthermore, we are grateful for the assistance and encouragement of our colleagues from the Geomatic Innovation Research Group, FGHT, UTM. Sincerest appreciation to Universiti Teknologi Malaysia for funding this research under Research University Grant,Vot number: Q.J130000.2527.11H08 and Q.J130000.2527.12H99. 


\section{REFERENCES}

Amos, M. J., \& Featherstone, W. E., 2003. Preparations for a new gravimetric geoid model of New Zealand, and some preliminary results. New Zealand Surveyor, 293, 9-20.

Childers, V., Bell, R., \& Brozena, J., 1999. Airborne gravimetry: An investigation of filtering. Geophysics, 64(1), 61-69. http://doi.org/doi:10.1190/1.1444530

Featherstone, W. E. , 2002. Report of IAG Special Study Group 3.177 Synthetic Modelling of the Earth's Gravity Field (1999-2001). Report of the IAG, 3, 163-171.a

Forsberg, R., Olesen, A., \& Keller, K., 1999.Airborne gravity survey of the north Greenland Shelf 1998. Kort-og Matrikelstyrelsen.

Heck, B., 1990. An evaluation of some systematic error sources affecting terrestrial gravity anomalies.Bulletin Géodésique, 64(1), 88-108.

Hughes C.W., Bingham R.J ,2008. An Oceanographer's Guide

to GOCE and the Geoid. Ocean Sci. 4: 15-29.

JUPEM,2003.Airborne gravity survey and geoid determination

project for Peninsular Malaysia, Sabah and Sarawak, Contract JUPEM-T04/2002, Final Report, Seksyen Geodesi, Bahagian Pemetaan.

JUPEM,2014. The Conduct of airborne gravity and magentic survey over selected area near the international; maritime boundary offshore of Sabah and Sarawak, Phase 1 (2014), Contract JUPEM-T03/2013,Final Report, Bahagian Ukur Geodetik.

JUPEM, 2015. The Conduct of airborne gravity and magnetic survey over selected area near the international maritime boundary offshore of Sabah and Sarawak, Phase II (2015), Contract JUPEM-T-24/204.

Kiahmehr, R., 2006. A hybrid precise gravimetric geoid model for Iran based on recent GRACE and SRTM data and the least squares modifications of Stokes's formula. Earth,32(1),7-23. Retrieved from http://www.geophysics.ut.ac.ir/JournalData/1385A/Kiamehr.p df

Marco Talone , Marco Meloni, Josep L. Pelegri, Miquel Rosell-

Fieschi and Rune Flobergaghen, 2014.Evoulution of geoids in recent years and its impact on oceanography

Mayer-Gürr, T., 2007. ITG-Grace03s: The latest GRACE gravity field solution computed in Bonn, (October 2007).

Olesen, A. V., \& Forsberg, R. , 2007. Airborne scalar gravimetry for regional gravity field mapping and geoid determination. Harita Dergisi.
Pavlis, N. K., Holmes, S. a., Kenyon, S. C., \& Factor, J. K. ,2008. An earth gravitational model to degree 2160: EGM2008. Presented at the 2008 General Assembly of the European Geosciences Union, Vienna, Austria, April 13-18, 84(1), 2-4. Retrieved from http://earthinfo.nga.mil/GandG/wgs84/gravitymod/egm2008/README_ WGS84_2.pdf

Reigber, C., Schwintzer, P., \& Lühr, H., 1999. The CHAMP geopotential mission. Boll. Geof. Teor. Appl,40, 285-289. Reigber, C., Lühr, H., \& Schwintzer, P.,2000. Status of the CHAMP mission. In Towards an Integrated Global Geodetic Observing System (IGGOS) (pp. 63-65). Springer Berlin Heidelberg.

Reigber, C., Schwintzer, P., Stubenvoll, R., Schmidt, R., Flechtner, F., Meyer, U., \& Zhu, S. Y., 2006.A high resolution global gravity field model combining CHAMP and GRACE satellite mission and surface data: EIGEN-CG01C. Geoforschungszentrum.

Rodríguez-Caderot, G., Lacy, M. C., Gil, A. J., \& Blazquez, B.,2006. Comparing recent geopotential models in Andalusia (Southern Spain). Studia Geophysica et Geodaetica, 50(4), 619-631.

Rummel, R., Balmino, G., Johannessen, J., Visser, P. N. A. M., \& Woodworth, P., 2002. Dedicated gravity field missions-principles and aims.Journal of Geodynamics, 33(1), 3-20.

Sadiq, M., \& Ahmad, Z. ,2009. On the selection of optimal global geopotential model for geoid modeling: A case study in Pakistan. Advances in Space Research, 44(5), 627639. http://doi.org/10.1016/j.asr.2009.05.004

Sokolov, A. V. , 2011. High accuracy airborne gravity measurements. Methods and equipment. IFAC Proceedings Volumes (IFAC-PapersOnline), 18(PART 1), 1889-1891. http://doi.org/10.3182/20110828-6-IT-1002.01892

Sulaiman, S. A., 2016. Gravimetric geoid model. Universiti Teknologi Mara.

Yi, W., \& Rummel, R., 2014. A comparison of GOCE gravitational models with EGM2008. Journal of Geodynamics, 73, 14-22. http://doi.org/10.1016/j.jog.2013.10.004

Zheleznyak L.K. , 2002. Russian marine gravimeter. Using gravity technologies in geophysics, Vol. 1. pp. 14-21. 
The International Archives of the Photogrammetry, Remote Sensing and Spatial Information Sciences, Volume XLII-4/W1, 2016 International Conference on Geomatic and Geospatial Technology (GGT) 2016, 3-5 October 2016, Kuala Lumpur, Malaysia

\section{APPENDIX}

THE LIST OF THE 130 POINTS USED FOR THE ASSESSMENT OF MARINE GEOID UNDULATIONS BETWEEN GGMS AND AIRBORNE GRAVITY DATA

\begin{tabular}{|c|c|c|c|c|c|c|c|}
\hline & Latitude & Longitude & AIRBORNE & EGM 2008 & $\begin{array}{c}\text { ITG-GRACE } \\
2010 \mathrm{~s}\end{array}$ & $\begin{array}{c}\text { ITG-GOCE } \\
\text { 02s }\end{array}$ & $\begin{array}{c}\text { AIUB } \\
\text { CHAMP 03s } \\
\end{array}$ \\
\hline 1 & 1.25 & 119.25 & 54.596 & 54.75537 & 55.4136 & 55.67394 & 54.3899 \\
\hline 2 & 1.5 & 119.25 & 55.173 & 55.28564 & 55.16727 & 55.11926 & 54.1808 \\
\hline 3 & 1.5 & 119.5 & 54.068 & 54.18852 & 54.53846 & 54.199 & 53.4512 \\
\hline 4 & 1.75 & 119.25 & 53.921 & 53.83353 & 54.82978 & 54.44681 & 53.4837 \\
\hline 5 & 1.75 & 119.5 & 53.573 & 53.66774 & 54.07631 & 53.61763 & 52.8645 \\
\hline 6 & 1.75 & 119.75 & 54.371 & 54.51857 & 53.9119 & 53.64043 & 53.5828 \\
\hline 7 & 2 & 119.5 & 52.887 & 52.93668 & 53.76737 & 53.36181 & 52.2125 \\
\hline 8 & 2 & 119.75 & 53.545 & 53.6536 & 53.52032 & 53.45576 & 52.9613 \\
\hline 9 & 3 & 109.5 & 26.217 & 26.33376 & 25.57608 & 26.49298 & 25.7647 \\
\hline 10 & 3.5 & 109.75 & 25.992 & 26.14231 & 25.33658 & 26.28298 & 25.7937 \\
\hline 11 & 3.5 & 110 & 26.956 & 27.10744 & 26.62602 & 27.12438 & 26.8338 \\
\hline 12 & 3.5 & 110.25 & 27.894 & 28.06517 & 27.94675 & 27.98627 & 27.8598 \\
\hline 13 & 3.75 & 109.5 & 24.551 & 24.70645 & 23.75504 & 24.82092 & 24.3731 \\
\hline 14 & 3.75 & 109.75 & 25.467 & 25.61858 & 24.79991 & 25.78482 & 25.371 \\
\hline 15 & 3.75 & 110 & 26.414 & 26.58105 & 26.01786 & 26.6586 & 26.3833 \\
\hline 16 & 6.5 & 109 & 16.161 & 16.30745 & 15.92407 & 16.3433 & 15.4353 \\
\hline 17 & 6.5 & 109.25 & 17.086 & 17.24093 & 16.78504 & 17.18762 & 16.1689 \\
\hline 18 & 6.5 & 109.5 & 17.644 & 17.74512 & 17.70967 & 18.01564 & 16.7948 \\
\hline 19 & 6.5 & 109.75 & 18.221 & 18.33638 & 18.65469 & 18.86641 & 17.5418 \\
\hline 20 & 6.5 & 110 & 19.413 & 19.56407 & 19.56666 & 19.76585 & 18.5843 \\
\hline 21 & 6.75 & 109 & 15.583 & 15.74164 & 15.20608 & 15.84136 & 14.954 \\
\hline 22 & 6.75 & 109.25 & 16.373 & 16.51793 & 16.12619 & 16.5448 & 15.6332 \\
\hline 23 & 6.75 & 109.5 & 16.777 & 16.92077 & 17.11635 & 17.18425 & 16.1386 \\
\hline 24 & 6.75 & 109.75 & 17.419 & 17.54866 & 18.11835 & 17.87405 & 16.7773 \\
\hline 25 & 6.75 & 110 & 18.612 & 18.74397 & 19.06864 & 18.70623 & 17.7925 \\
\hline 26 & 6.75 & 115.25 & 39.967 & 40.18163 & 39.62065 & 40.12424 & 39.7443 \\
\hline 27 & 6.75 & 115.5 & 42.448 & 42.65544 & 41.33411 & 41.89402 & 42.0803 \\
\hline 28 & 6.75 & 115.75 & 44.42 & 44.62396 & 43.04049 & 43.61766 & 43.9589 \\
\hline 29 & 6.75 & 116 & 45.58 & 45.78489 & 44.71131 & 45.28551 & 45.1203 \\
\hline 30 & 6.75 & 116.25 & 46.345 & 46.51129 & 46.31785 & 46.85542 & 45.8478 \\
\hline 31 & 7 & 109 & 14.952 & 15.12652 & 14.41094 & 15.23453 & 14.5048 \\
\hline 32 & 7 & 109.25 & 15.701 & 15.86222 & 15.39733 & 15.85451 & 15.1957 \\
\hline 33 & 7 & 109.5 & 16.075 & 16.21132 & 16.46108 & 16.37659 & 15.6848 \\
\hline 34 & 7 & 109.75 & 16.668 & 16.79236 & 17.52675 & 16.97319 & 16.2657 \\
\hline 35 & 7 & 110 & 17.753 & 17.87365 & 18.521 & 17.78389 & 17.164 \\
\hline 36 & 7 & 115.25 & 38.47 & 38.70292 & 38.67207 & 38.83792 & 37.9638 \\
\hline 37 & 7 & 115.5 & 40.975 & 41.16045 & 40.15915 & 40.4956 & 40.4867 \\
\hline 38 & 7 & 115.75 & 43.234 & 43.41638 & 41.66951 & 42.15944 & 42.6779 \\
\hline 39 & 7 & 116 & 44.646 & 44.84142 & 43.20761 & 43.80774 & 44.0245 \\
\hline 40 & 7 & 116.25 & 45.423 & 45.55412 & 44.77169 & 45.41204 & 44.7242 \\
\hline 41 & 7.25 & 109 & 14.339 & 14.51212 & 13.58589 & 14.54542 & 13.9896 \\
\hline
\end{tabular}


The International Archives of the Photogrammetry, Remote Sensing and Spatial Information Sciences, Volume XLII-4/W1, 2016 International Conference on Geomatic and Geospatial Technology (GGT) 2016, 3-5 October 2016, Kuala Lumpur, Malaysia

\begin{tabular}{|c|c|c|c|c|c|c|c|}
\hline 42 & 7.25 & 109.25 & 15.135 & 15.30221 & 14.63468 & 15.18378 & 14.7209 \\
\hline 43 & 7.25 & 109.5 & 15.652 & 15.78882 & 15.76319 & 15.70429 & 15.287 \\
\hline 44 & 7.25 & 109.75 & 16.252 & 16.45626 & 16.88091 & 16.30498 & 15.917 \\
\hline 45 & 7.25 & 110 & 16.998 & 17.14418 & 17.90914 & 17.14396 & 16.7425 \\
\hline 46 & 7.25 & 115.25 & 37.041 & 37.26626 & 37.88184 & 37.8099 & 36.3136 \\
\hline 47 & 7.25 & 115.5 & 39.109 & 39.30793 & 39.06048 & 39.16704 & 38.4682 \\
\hline 48 & 7.25 & 115.75 & 41.107 & 41.27911 & 40.32158 & 40.62442 & 40.6389 \\
\hline 49 & 7.25 & 116 & 42.662 & 42.79534 & 41.7059 & 42.18069 & 42.1695 \\
\hline 50 & 7.25 & 116.25 & 43.914 & 44.03029 & 43.23294 & 43.82074 & 43.0915 \\
\hline 51 & 7.25 & 118 & 51.206 & 51.42618 & 51.30697 & 52.0485 & 50.5595 \\
\hline 52 & 7.25 & 118.25 & 51.994 & 52.22212 & 51.66483 & 52.29276 & 51.0333 \\
\hline 53 & 7.5 & 109 & 13.728 & 13.91234 & 12.81344 & 13.85152 & 13.4028 \\
\hline 54 & 7.5 & 109.25 & 14.543 & 14.69783 & 13.91136 & 14.59317 & 14.1504 \\
\hline 55 & 7.5 & 109.5 & 15.311 & 15.42188 & 15.08078 & 15.21073 & 14.8331 \\
\hline 56 & 7.5 & 109.75 & 16.16 & 16.35353 & 16.22263 & 15.89458 & 15.637 \\
\hline 57 & 7.5 & 110 & 16.793 & 16.97705 & 17.26096 & 16.793 & 16.5633 \\
\hline 58 & 7.5 & 115.25 & 36.185 & 36.42265 & 37.24708 & 37.1584 & 35.3311 \\
\hline 59 & 7.5 & 115.5 & 37.577 & 37.75958 & 38.10801 & 38.12406 & 36.804 \\
\hline 60 & 7.5 & 115.75 & 39.398 & 39.51211 & 39.12724 & 39.28241 & 38.5931 \\
\hline 61 & 7.5 & 116 & 40.839 & 40.91919 & 40.37569 & 40.67721 & 40.113 \\
\hline 62 & 7.5 & 116.25 & 42.133 & 42.21014 & 41.8844 & 42.31236 & 41.3143 \\
\hline 63 & 7.5 & 118.25 & 51.127 & 51.32983 & 51.22478 & 51.37273 & 50.2891 \\
\hline 64 & 7.5 & 118.5 & 51.8 & 52.04058 & 51.60982 & 51.51561 & 50.9085 \\
\hline 65 & 7.75 & 115.25 & 36.124 & 36.30435 & 36.73077 & 36.8444 & 35.2131 \\
\hline 66 & 7.75 & 115.5 & 37.045 & 37.1666 & 37.33845 & 37.45564 & 36.059 \\
\hline 67 & 7.75 & 115.75 & 38.09 & 38.1643 & 38.17652 & 38.31245 & 37.2629 \\
\hline 68 & 7.75 & 116 & 39.309 & 39.38043 & 39.33247 & 39.51038 & 38.5076 \\
\hline 69 & 7.75 & 116.25 & 40.728 & 40.79458 & 40.83863 & 41.08059 & 39.8361 \\
\hline 70 & 7.75 & 118.25 & 50.118 & 50.34244 & 50.72216 & 50.49956 & 49.507 \\
\hline 71 & 7.75 & 118.5 & 50.677 & 50.87307 & 50.99593 & 50.60771 & 49.9506 \\
\hline 72 & 8 & 109 & 12.759 & 12.9644 & 11.72048 & 12.68786 & 12.2983 \\
\hline 73 & 8 & 109.25 & 13.224 & 13.3886 & 12.8472 & 13.62524 & 13.0192 \\
\hline 74 & 8 & 109.5 & 13.942 & 14.09685 & 13.98928 & 14.45928 & 13.7801 \\
\hline 75 & 8 & 109.75 & 15.02 & 15.13645 & 15.06022 & 15.32438 & 14.8792 \\
\hline 76 & 8 & 110 & 16.425 & 16.54703 & 16.01985 & 16.31463 & 16.2232 \\
\hline 77 & 8 & 115.25 & 36.419 & 36.54215 & 36.29384 & 36.72164 & 35.5823 \\
\hline 78 & 8 & 115.5 & 37.044 & 37.14072 & 36.76561 & 37.12106 & 36.078 \\
\hline 79 & 8 & 115.75 & 37.661 & 37.69987 & 37.5131 & 37.75756 & 36.7525 \\
\hline 80 & 8 & 116 & 38.389 & 38.41437 & 38.62317 & 38.76592 & 37.6055 \\
\hline 81 & 8 & 116.25 & 39.806 & 39.8601 & 40.12223 & 40.21234 & 38.8542 \\
\hline 82 & 8 & 118.25 & 49.188 & 49.37688 & 50.20295 & 49.86781 & 48.6752 \\
\hline 83 & 8 & 118.5 & 49.504 & 49.60573 & 50.33864 & 49.92514 & 48.7676 \\
\hline 84 & 8.25 & 109 & 12.232 & 12.42501 & 11.41634 & 12.19376 & 11.7403 \\
\hline 85 & 8.25 & 109.25 & 12.739 & 12.91614 & 12.51536 & 13.11934 & 12.4826 \\
\hline 86 & 8.25 & 109.5 & 13.425 & 13.56278 & 13.58809 & 13.9569 & 13.1604 \\
\hline 87 & 8.25 & 109.75 & 14.549 & 14.67794 & 14.56814 & 14.82635 & 14.1689 \\
\hline 88 & 8.25 & 110 & 15.869 & 16.03123 & 15.4452 & 15.80747 & 15.5202 \\
\hline 89 & 8.25 & 115.25 & 36.941 & 37.08556 & 35.92056 & 36.63973 & 35.8654 \\
\hline 90 & 8.25 & 115.5 & 36.978 & 37.0872 & 36.39127 & 37.01446 & 36.2953 \\
\hline 91 & 8.25 & 115.75 & 37.443 & 37.51174 & 37.13783 & 37.55301 & 36.7271 \\
\hline
\end{tabular}


The International Archives of the Photogrammetry, Remote Sensing and Spatial Information Sciences, Volume XLII-4/W1, 2016 International Conference on Geomatic and Geospatial Technology (GGT) 2016, 3-5 October 2016, Kuala Lumpur, Malaysia

\begin{tabular}{|c|c|c|c|c|c|c|c|}
\hline 92 & 8.25 & 116 & 38.246 & 38.23435 & 38.23001 & 38.40468 & 37.3426 \\
\hline 93 & 8.25 & 116.25 & 39.154 & 39.17204 & 39.68651 & 39.67634 & 38.4217 \\
\hline 94 & 8.5 & 109 & 11.689 & 11.87977 & 11.17087 & 11.68057 & 11.119 \\
\hline 95 & 8.5 & 109.25 & 12.203 & 12.38699 & 12.21946 & 12.52984 & 11.8986 \\
\hline 96 & 8.5 & 109.5 & 12.795 & 12.96919 & 13.20009 & 13.29819 & 12.4703 \\
\hline 97 & 8.5 & 109.75 & 13.699 & 13.83022 & 14.07097 & 14.10801 & 13.2925 \\
\hline 98 & 8.5 & 110 & 14.779 & 14.85991 & 14.85382 & 15.04191 & 14.508 \\
\hline 99 & 8.5 & 115.25 & 36.673 & 36.82291 & 35.62187 & 36.52189 & 35.8257 \\
\hline 100 & 8.5 & 115.5 & 37.2 & 37.30645 & 36.20606 & 37.03481 & 36.3612 \\
\hline 101 & 8.5 & 115.75 & 37.594 & 37.65256 & 37.01366 & 37.58636 & 36.8833 \\
\hline 102 & 8.5 & 116 & 38.202 & 38.26389 & 38.08518 & 38.31372 & 37.5529 \\
\hline 103 & 8.5 & 116.25 & 39.044 & 39.12885 & 39.43521 & 39.36606 & 38.5019 \\
\hline 104 & 8.75 & 109 & 11.132 & 11.35306 & 10.85775 & 11.11207 & 10.4714 \\
\hline 105 & 8.75 & 109.25 & 11.608 & 11.79309 & 11.84773 & 11.87571 & 11.2567 \\
\hline 106 & 8.75 & 109.5 & 12.208 & 12.34409 & 12.73501 & 12.55173 & 11.7481 \\
\hline 107 & 8.75 & 109.75 & 13.124 & 13.23205 & 13.50128 & 13.27382 & 12.4268 \\
\hline 108 & 8.75 & 110 & 13.992 & 14.12741 & 14.19692 & 14.13828 & 13.5007 \\
\hline 109 & 8.75 & 112.75 & 26.335 & 26.52814 & 25.93124 & 26.49467 & 25.702 \\
\hline 110 & 8.75 & 113 & 27.242 & 27.48386 & 27.00661 & 27.51365 & 26.7526 \\
\hline 111 & 8.75 & 115.25 & 35.869 & 36.04829 & 35.41484 & 36.36479 & 35.6177 \\
\hline 112 & 8.75 & 115.5 & 36.749 & 36.90553 & 36.17922 & 37.10785 & 36.3067 \\
\hline 113 & 8.75 & 115.75 & 37.852 & 37.91401 & 37.06921 & 37.73872 & 37.0785 \\
\hline 114 & 8.75 & 116 & 38.47 & 38.55388 & 38.08987 & 38.36158 & 37.9619 \\
\hline 115 & 8.75 & 116.25 & 39.565 & 39.66272 & 39.25812 & 39.16214 & 38.8291 \\
\hline 116 & 9 & 109 & 10.544 & 10.79153 & 10.37259 & 10.49634 & 9.81985 \\
\hline 117 & 9 & 109.25 & 11.066 & 11.26546 & 11.31685 & 11.21844 & 10.5583 \\
\hline 118 & 9 & 109.5 & 11.525 & 11.67477 & 12.13463 & 11.83491 & 11.0022 \\
\hline 119 & 9 & 109.75 & 12.445 & 12.55282 & 12.82446 & 12.48638 & 11.6384 \\
\hline 120 & 9 & 110 & 13.313 & 13.36243 & 13.45701 & 13.28098 & 12.6522 \\
\hline 121 & 9 & 112.5 & 25.05 & 24.90229 & 24.30062 & 24.8941 & 24.411 \\
\hline 122 & 9 & 112.75 & 25.813 & 25.74825 & 25.11479 & 25.80926 & 25.2195 \\
\hline 123 & 9 & 113.5 & 29.026 & 29.11852 & 28.61132 & 29.19352 & 28.4135 \\
\hline 124 & 9 & 113.75 & 30.152 & 30.25427 & 29.87513 & 30.26972 & 29.524 \\
\hline 125 & 9 & 114.5 & 32.926 & 33.00073 & 32.77926 & 32.88821 & 32.5061 \\
\hline 126 & 9 & 115.25 & 35.708 & 35.90085 & 35.29147 & 36.17533 & 35.4259 \\
\hline 127 & 9 & 115.5 & 36.902 & 36.97301 & 36.24634 & 37.1589 & 36.2493 \\
\hline 128 & 9 & 115.75 & 37.952 & 37.91849 & 37.20444 & 37.89045 & 37.1983 \\
\hline 129 & 9 & 116 & 38.546 & 38.55429 & 38.13475 & 38.42817 & 38.2145 \\
\hline 130 & 9 & 116.25 & 39.558 & 39.50601 & 39.06176 & 38.97735 & 38.9924 \\
\hline
\end{tabular}

